\title{
Terzan 5: a pristine fragment of the Bulge
}

\author{
Francesco R. Ferraro \\ Dipartimento di Fisica e Astronomia, Università di Bologna (Italy) \\ email: francesco.ferraro3@unibo.it
}

\begin{abstract}
We have discovered that Terzan 5, a stellar system in the Galactic Bulge, harbors two stellar populations with different iron content $(\Delta[\mathrm{Fe} / \mathrm{H}] \sim 0.5$ dex $)$ and possibly different ages. Moreover, the observed chemical patterns significantly differ from those observed in any known genuine globular cluster. These evidences demonstrate that, similarly to $\omega$ Centauri in the Halo, Terzan 5 is not a globular cluster, but a stellar system that was able to retain the gas ejected by violent supernova explosions. Moreover the striking chemical similarity with the Bulge stars suggests that Terzan 5 could be the relic of one of the massive clumps that contributed (through strong dynamical interactions with other pre-formed and internally-evolved sub-structures) to the formation of the Galactic Bulge.
\end{abstract}

Keywords. Stars: abundances - Globular clusters: individual (Terzan 5)

There is now a great deal of evidence for a significant spread in the abundance patterns of a few light-elements (as Na, O, Al, etc.) in Galactic globular clusters (GCs; see Carretta et al. 2010). This suggests that GC formation may have been more complex than previously thought. The multiple sequences observed in a few GCs are interpreted as the photometric manifestation of this phenomenon and of the connected variations in the $\mathrm{He}$ abundance (Piotto 2009 and references therein). However, GCs still are the best approximations of simple stellar populations we have in Nature, with their striking homogeneity in terms of the iron content still holding and indicating that their stellar populations formed in a parent potential well which was too shallow to retain the high-velocity gas ejected by violent supernova (SN) explosions. To date only two major exceptions to this rule are known: $\omega$ Centauri (Norris \& Da Costa 1995) in the Galactic Halo, and Terzan 5 (Ferraro et al. 2009) in the Bulge.

The case of Terzan 5 - Thanks to high-resolution NIR imaging obtained with the multiconjugate adaptive-optics demonstrator MAD@VLT, we revealed the presence of two distinct red clumps in Terzan 5 (Fig.1a), and from NIR spectroscopy with NIRSPEC@Keck we demonstrated that the two stellar populations are characterized by very different iron contents: $[\mathrm{Fe} / \mathrm{H}]=-0.2$ and +0.3 (Ferraro et al. 2009). In addition, follow-up spectroscopy (Origlia et al. 2011) revealed no evidence of the Al-O anti-correlation commonly found in GCs and showed that the metal-poor component has $[\alpha / \mathrm{Fe}]=+0.34$, suggesting that it formed early and quickly from a gas mainly polluted by a huge amount of SNeII, while the metal-rich population has $[\alpha / \mathrm{Fe}]=+0.03$, indicating that it experienced additional enrichment from both SNII and SNIa ejecta on longer timescales.

These observational facts clearly demonstrate that Terzan 5 is far from being a genuine GC. Instead, they can be naturally explained within a self-enrichment scenario, where the proto-Terzan 5 was much more massive in the past than today (its current mass being $10^{6} M_{\odot}$; Lanzoni et al. 2010) and experienced two main and relatively short episodes of star formation with a separation of a few Gyr (a timescale which is much longer than assumed to account for multiple populations in genuine GCs): Terzan 5 was therefore able to retain the SN ejecta and gave rise to two distinct populations with different iron content. The high number of SNeII would have also produced a large population of 

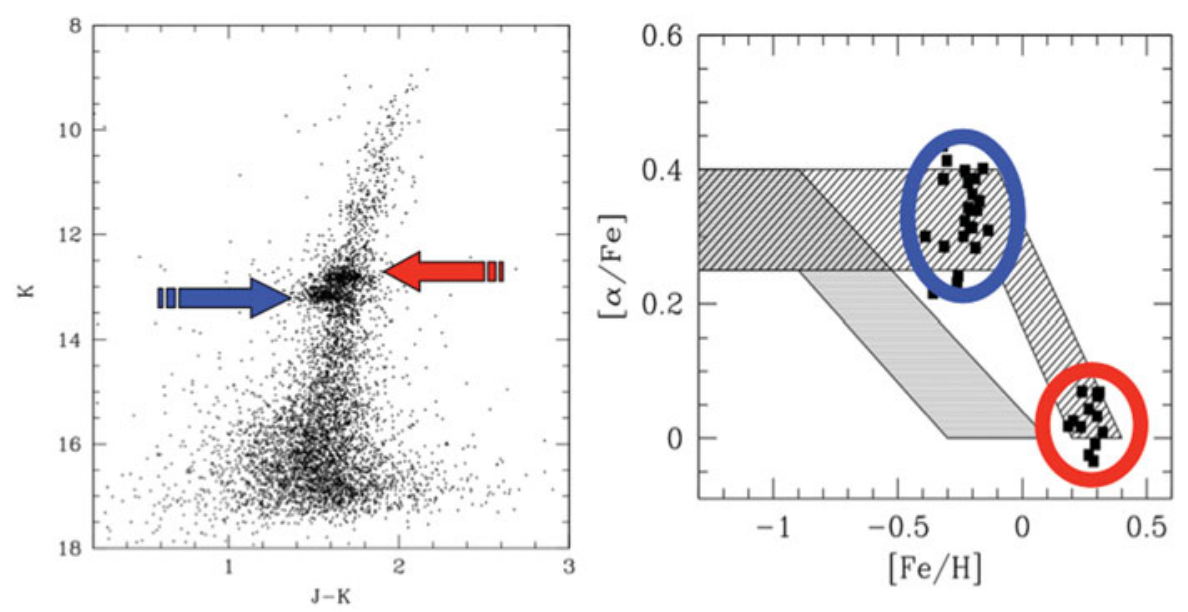

Figure 1. left panel: The two red clumps discovered in Terzan 5 thanks to $K$ and $J$ MAD observations. right panel: $[\alpha / \mathrm{Fe}]$ and $[\mathrm{Fe} / \mathrm{H}]$ ratios for a sample of giants in Terzan 5 (with the two populations highlighted by the blue and red circles, according to the left panel), compared to those of the Galactic Bulge (shaded region) and Halo+Disk giants (grey region).

neutron stars, most of which would have been retained within the deep potential well of the system. Then the high collision rate of Terzan 5 (the highest among all GCs, Lanzoni et al. 2010) could have favored the formation of binary systems containing neutron stars and promoted the recycling process that finally generated the huge population of millisecond pulsars now observed in Terzan 5 (which is the largest population ever detected in any stellar system; Ransom et al. 2005).

Indeed, Terzan 5 has all the characteristics expected for a system which had an original mass larger than a few $10^{7}-10^{8} M_{\odot}$ and experienced a complex evolutionary history. Moreover its metal content and $\alpha$-enhancement very closely resemble those of the Bulge stars (see Fig.1b; e.g. Rich et al. 2007; Zoccali et al. 2008). These striking similarities with the Bulge population strongly suggest that, at odds with $\omega$ Centauri that was probably accreted from outside the Galaxy, Terzan 5 formed and evolved within its current environment, the Bulge. Indeed these two stellar systems could have shared a common origin and evolutionary history, and Terzan 5 might well be the fossil fragment of a larger substructure that contributed to form the Galactic Bulge via strong dynamical interactions with other similar systems (e.g. Immeli et al. 2004).

This research is part of the project Cosmic-Lab funded by the European Research Council (under contract ERC-2010-AdG-267675).

\section{References}

Carretta, E., et al. 2009, A\& $A, 505,117$

Ferraro, F. R., et al. 2009, Nature, 462, 483

Immeli, A., et al. 2004, A\&BA, 413, 547

Lanzoni, B., et al. 2010, ApJ, 717, 653

Norris, J. E. \& Da Costa, G. S. 1995, ApJ, 441, L81

Origlia, L., et al. 2011, ApJ, 726, 20

Piotto, G. 2009, IAUS, 258, 233

Ransom, S., et al. 2005, Science, 307, 892

Rich, M., et al. 2007, ApJ, 665, 119

Zoccali, M., et al. 2008, A\&SA, 486, 177 